\title{
RM41
}

\section{DelineatingThickness Distribution Based on Snesim Simulation}

C. Pardosi* (Bandung Institute of Technology (ITB)), D. Hapsari (Bandung
Institute of Technology (ITB)), S. Winardhi (Bandung Institute of
Technology (ITB)) \& A. Syahputra (Bandung Institute of Technology (ITB))

\section{SUMMARY}

This Paper presents an approach in estimating sand distribution in a fluvial environment by using gradient of spectral decomposition as the soft constraint data. Training image used in the simulation is fluvial model with certain pattern and probability of sand and shale. As soft constraint, spectral decomposition is used to identify the thickness of sand within the area, and also to determine shale area. Then validators are used to analyze the reliability of MPS. 


\section{Introduction}

Multi Point Statistic (MPS) is commonly used in reservoir modeling. In this study, SNESIM algorithm is used to gain sandstone distribution in a reservoir. The data used in this study are, sand thickness in each well (hard data), gradient of spectral decomposition (used to calculate probability and as soft constraint), and training image of fluvial geological environment. In this case, the limited amount of hard data caused difficulties in modeling variogram to construct continuous pattern, so the variogram cannot be used in structure delineating. For that reason training image substitutes variogram as geological heterogeneity parameter in MPS. Several simulations were done to obtain the mean distribution as the final result. The final result itself represents the percentage of sandstone probability for validating wells that are excluded in the simulations.

\section{Method}

Algorithm used in this simulation is Single Normal Equation Simulation (SNESIM) to verify that this algorithm only involves a single normal equation to receive conditional probability (Strebelle, 2000). Unlike other algorithms that use variogram to simulate continuous variable, SNESIM utilize training image and simulate discrete variable. MPS uses training image as a reference to delineate the structure because the main idea is pattern recognition from training image. Training image itself can be defined as a conceptual model from lithofacies of a reservoir. The soft constraint used should be a probability.

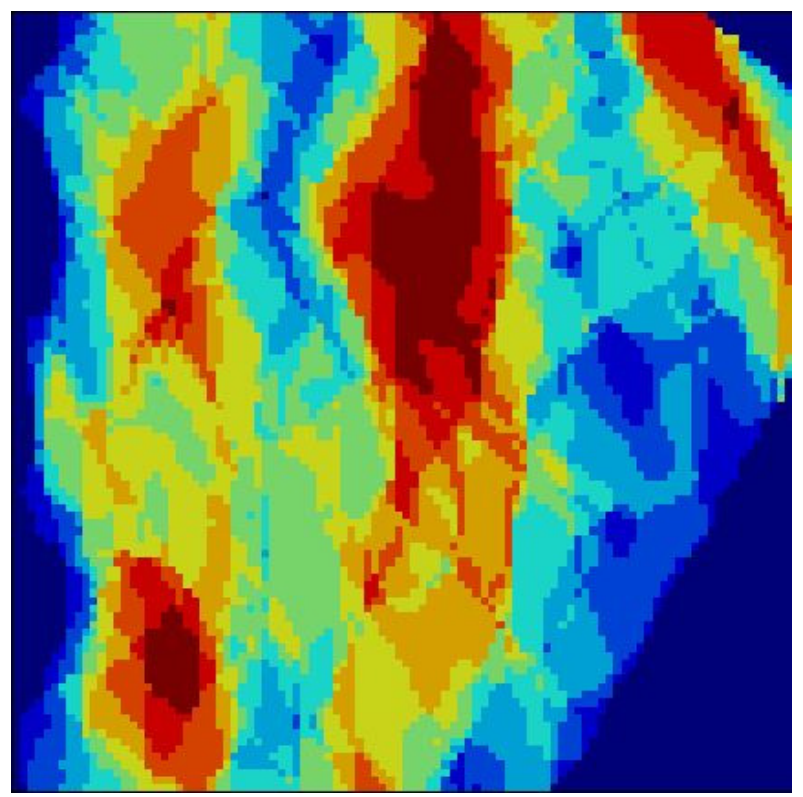

Figure 1 The mean distribution of sandstone.

\section{Conclusions}

Based on this study, it can be concluded that MPS can create a reliable sandstone distribution map. This is proven by the validator well tested on the final result that matched with our definition of the validator.

\section{References}

Deutsch, C.V. and Tran, T.T. [2002] FLUVSIM: A Program for Object-Based Stochastic Modeling of Fluvial Depositional Systems. Computers and Geosciences, 28.

Putri, S.A. [2011] Application of Multi-Point Geostatistics for Reservoir Sandstone Delineation: Hard Data and Soft Constraint Testing, Final Project, Institut Teknologi Bandung. 